\title{
Home-based hand rehabilitation after chronic stroke: Randomized, controlled single-blind trial comparing the MusicGlove with a conventional exercise program
}

\author{
Daniel K. Zondervan, PhD; ${ }^{*}$ Nizan Friedman, PhD; ${ }^{2}$ Enoch Chang, MD; ${ }^{3}$ Xing Zhao, MD; ${ }^{3}$ Renee Augsburger, \\ OTR/L; ${ }^{4}$ David J. Reinkensmeyer, PhD; ${ }^{1-3,5}$ Steven C. Cramer, MD ${ }^{3,5-6}$ \\ Departments of ${ }^{1}$ Mechanical and Aerospace Engineering, ${ }^{2}$ Biomedical Engineering, and ${ }^{3}$ Physical Medicine and Rehabilita- \\ tion, University of California at Irvine, Irvine, $C A,{ }^{4}$ Rehabilitation Services, University of California at Irvine Medical Center, \\ Irvine, CA; Departments of ${ }^{5}$ Anatomy and Neurobiology and ${ }^{6}$ Neurology, University of California at Irvine, Irvine, CA
}

\begin{abstract}
Individuals with chronic stroke have limited options for hand rehabilitation at home. Here, we sought to determine the feasibility and efficacy of home-based MusicGlove therapy. Seventeen participants with moderate hand impairment in the chronic phase of stroke were randomized to 3 wk of home-based exercise with either the MusicGlove or conventional tabletop exercises. The primary outcome measure was the change in the Box and Blocks test score from baseline to 1 mo posttreatment. Both groups significantly improved their Box and Blocks test score, but no significant difference was found between groups. The MusicGlove group did exhibit significantly greater improvements than the conventional exercise group in Motor Activity Log Quality of Movement and Amount of Use scores 1 mo posttherapy ( $p=0.007$ and $p=0.04$, respectively). Participants significantly increased their use of MusicGlove over time, completing 466 gripping movements per day on average at study end. MusicGlove therapy was not superior to conventional tabletop exercises for the primary end point but was nevertheless feasible and led to a significantly greater increase in self-reported functional use and quality of movement of the impaired hand than conventional home exercises.
\end{abstract}

Clinical Trial Registration: ClinicalTrials.gov; "Influence of Timing on Motor Learning”; NCT01769326;

https://clinicaltrials.gov/ct2/show/NCT01769326

Key words: hand impairment, hand therapy, home therapy, Motor Activity Log, music therapy, randomized controlled trial, rehabilitation, stroke, task-specific rehabilitation, virtual reality.

\section{INTRODUCTION}

Hand impairment after stroke contributes substantially to disability in the United States and around the world [1]. Intensive movement practice can reduce hand impairment [2-6], but issues such as cost and access may limit the dose of rehabilitation exercise delivered one-onone with a therapist. Because of these and other factors, most individuals do not perform the large number of exercise repetitions required during therapy to maximize recovery [7-8]. Home-based rehabilitation programs may be prescribed after stroke with the intent to increase the amount of rehabilitation exercise individuals perform. However, the most common approach to home-based hand therapy is following a printed handout of exercises. This approach is often not motivating and thus is associated with low compliance and high dropout rates [9-13].

\footnotetext{
Abbreviations: ANOVA $=$ analysis of variance, $\mathrm{AOU}=$ Amount of Use, ARAT = Action Research Arm Test, MAL = Motor Activity Log, QOM = Quality of Movement.

*Address all correspondence to Daniel K. Zondervan, PhD; Department of Mechanical and Aerospace Engineering, UC Irvine, 2402 Calit2 Building, Irvine, CA 92697; 562716-7277. Email: dkzondervan@gmail.com

http://dx.doi.org/10.1682/JRRD.2015.04.0057
} 
To address this problem, other types of home-based rehabilitation programs for the hand have been proposed. For example, one pilot study explored a modified form of constraint-induced movement therapy performed under the supervision of a nonprofessional coach in the home and found similar benefits to the same program performed with a trained therapist in a clinic [14]; a larger study using this protocol found that home-based constraint-induced movement therapy led to significantly greater self-reported use of the impaired limb than conventional therapy [15]. Another common approach is telerehabilitation, which allows a therapist to guide therapy remotely [16]. While this approach is gaining popularity, a recent Cochrane systematic review of 10 trials with 933 total participants found limited evidence to support its use and no studies that examined its costeffectiveness [17]. Other approaches to home-based hand rehabilitation include functional electrical stimulation [18], computer gaming with custom devices [19-21], and musicbased therapy [22]. However, despite the variety of options, few home-based programs have been tested in controlled studies [23]. Further, it is still unclear which methods are the most effective and efficient means of providing an increased dose of rehabilitation, though the use of computer games and music has been found to be highly motivating [20,24-26].

We developed the MusicGlove, an instrumented glove with sensors on each of the fingertips and the lateral aspect of the index finger. The MusicGlove requires the user to practice functional gripping movements by touching the sensor on the tip of the thumb to one of the other five sensors in time with music through a video game that displays scrolling notes on a screen (Figure 1). In previous pilot studies performed in a clinical setting, we found that the MusicGlove motivated individuals with chronic stroke to perform hundreds of functional gripping movements during a 30 min training session and that exercise with the device led to a significantly greater improvement in hand grasping ability, measured with the Box and Blocks test, than a time-matched dose of conventional tabletop therapy performed with a rehabilitation therapist [27-28]. The individuals who used the MusicGlove also reported that the exercise was more motivating than conventional therapy and expressed interest in using the device to exercise at home. An important question, therefore, was whether self-guided exercise with the MusicGlove performed at home is feasible and improves hand function compared with conventional home therapy.

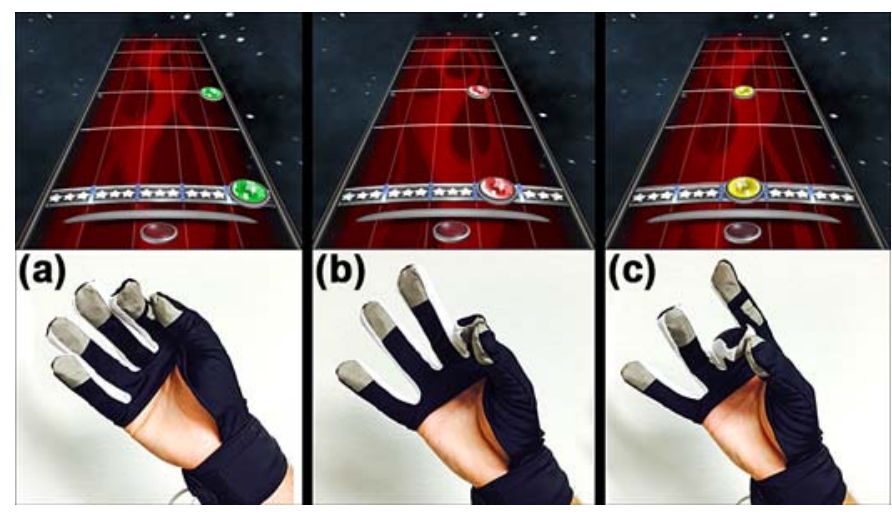

Figure 1.

MusicGlove device used in study. Users are visually cued by scrolling notes on screen (top) to make specific grips in time with popular songs, similar to the video game Guitar Hero. Grips include (a) key pinch grip; (b) pincer grip; and (c) finger-thumb opposition with second, third, and fourth fingers. During gameplay, the user must complete the cued grip when a colored note passes over the starred strip shown at bottom of the game screen (time window of about $800 \mathrm{~ms}$ ). If the user is successful, the colored note disappears, providing visual feedback. If the user is unsuccessful, a beep is played, providing auditory feedback.

To address this question, we performed a randomized, controlled single-blind trial that compared homebased training with the MusicGlove to home-based conventional tabletop training directed using a pamphlet of hand exercises. We hypothesized that the participants in the MusicGlove therapy group would improve their hand function more than the participants in the conventional therapy group when assessed 1 mo after treatment.

\section{METHODS}

\section{Study Design and Inclusion Criteria}

This study was a home-based randomized controlled trial that compared self-guided exercise with the MusicGlove to self-guided conventional tabletop therapy for individuals in the chronic phase of stroke. The study protocol was approved by University of California at Irvine's Institutional Review Board and registered at ClinicalTrials.gov (\#NCT01769326). All participants provided informed consent. Inclusion criteria were one or more strokes with onset $>6$ mo prior to the study, Box and Blocks score of at least 1 , ability to understand the instructions for the home exercise programs, and age $<75$ yr. 
Exclusion criteria were severe pain in the affected upper limb measured as a score $>5$ in the visual analog pain scale, severe tone in the affected upper limb measured as a score $>3$ in the Ashworth Scale of Spasticity for the wrist and elbow, severe loss of sensation of the affected upper limb measured as a score $<10$ in the Fugl-Meyer Sensory Examination, concurrent severe medical problems, visual deficits, severe neglect or apraxia, and enrollment in other upper-limb therapy studies.

\section{Outcome Measures}

All assessments were performed at the University of California at Irvine by two blinded, experienced evaluators. The primary end point was the change in Box and Blocks score, which measures how many blocks a subject can pick up and place in a box in $60 \mathrm{~s}$ [29-30], from baseline to 1 mo posttherapy. Secondary end points included changes in the Quality of Movement (QOM) and Amount of Use (AOU) subscales of the Motor Activity Log (MAL) [31-32], which is a structured interview that asks subjects to rate how well and how much they use their upper limb in performing 30 activities of daily living outside of the laboratory; the Nine Hole Peg test [33], which measures how many pegs a subject can put in and remove from holes in $60 \mathrm{~s}$; and the Action Research Arm Test (ARAT), which assesses the ability to manipulate various sized objects with the impaired arm and hand [30,34].

The following tests were also administered at baseline to characterize subjects: the Geriatric Depression Scale [35]; the upper-limb section of the Fugl-Meyer score [36], which measures impairment of the hemiparetic upper limb; the National Institutes of Health stroke scale [37]; and the modified Ashworth spasticity scale for the wrist [38]. These measures have established excellent sensitivity and reliability.

\section{Interventions}

All participants were invited for an initial assessment to confirm that they met the inclusion criteria and to establish their baseline characteristics. Those who met the inclusion criteria were instructed to return $1 \mathrm{wk}$ later for a second assessment to measure whether they had a stable baseline. Repeating the baseline assessments also accounted for familiarization or learning effects that could distort gains from true recovery [39-40]. At this time, the participants were randomly assigned to either the MusicGlove therapy group or conventional therapy group (i.e., the control group). To ensure matched levels of impairment between groups, subjects were first stratified by their second Box and Blocks baseline score (0-30 or 30-60) and then randomized by alternating block allocation, a technique referred to as adaptive randomization [41].

Participants randomized to the MusicGlove therapy group were given a MusicGlove device and a laptop with the software preinstalled. The MusicGlove devices used in this study were manufactured by Flint Rehabilitation Devices. They received a 15 min instruction on how to put on and use the device and how to operate the accompanying software. Participants in the conventional therapy group were given a booklet of tabletop exercises for home therapy of the hand developed by experienced occupational therapists and implemented in a prior clinical trial [42] and were instructed on how to correctly perform each hand exercise (see Appendix, available online only). Both groups were asked to perform self-guided therapy for at least $3 \mathrm{~h} / \mathrm{wk}$ over at least three sessions per week, for three consecutive weeks, for a total of $9 \mathrm{~h}$ of therapy; such a dose had been found previously to be sufficient to induce significant improvements in hand movement ability [8,43-45]. Both groups were also asked to manually record the amount of time they spent performing their self-guided therapy on a written exercise log. The laptops provided with the MusicGlove also recorded the number of grips completed with the device. The participants were contacted by a research therapist or nurse at least once a week to address any potential technical difficulties and to ensure there were no adverse health effects from the prescribed therapy.

After the 3 wk exercise period, the participants returned for posttherapy assessments. At this assessment, participants in the MusicGlove group returned the device, and participants in the conventional therapy group were instructed to discontinue their exercises. Participants then returned 1 mo later for follow-up assessments.

\section{Crossover}

As part of a secondary aim, participants in the conventional therapy group were given a MusicGlove device and laptop and instructed on how to use it after the 1 mo posttherapy assessment. These participants were asked to repeat the same therapy regimen ( $3 \mathrm{~h} / \mathrm{wk}$ for $3 \mathrm{wk}$ ) using the MusicGlove to exercise at home. At the end of this crossover exercise period, these participants returned for posttherapy assessments and returned the device. They again returned 1 mo later for follow-up assessments. 


\section{Data Analysis}

We anticipated an effect size of MusicGlove therapy of 1.11 based on an independent samples $t$-test immediately after therapy in our initial pilot study [27]. Thus, 11 participants in each group would provide an 80 percent chance to demonstrate a significant difference between the MusicGlove and control therapies at the 0.05 significance level (one-tailed).

Two-tailed Student $t$-tests (for continuous data) and Fisher exact tests (for categorical data) were used to compare baseline measures between the two treatment groups. For each of the outcome measures, if there was no significant difference in the group mean from the first to the second baseline, we calculated the individual changes at each follow-up assessment as the difference from the average of the two baseline values. If there was a difference in the group mean between baseline assessments for a particular outcome measure, indicating a familiarization or learning effect, individual changes were calculated from the second baseline value only. Note, the MAL was assessed at the first baseline assessment only, and thus changes in MAL scores were calculated from this single baseline measurement. To account for floor effects, the Nine Hole Peg test scores were measured in pegs placed and removed in $1 \mathrm{~min}$ [46].

The resulting data did not deviate significantly from normality for any of the outcome measures at all assessments (Lilliefors test). Thus, a one-tailed $t$-test was used to test for a significant difference in the primary end point between the two training conditions at 1 mo posttherapy. We elected to use a one-tailed test since our primary goal was to determine whether MusicGlove therapy was an improvement over conventional therapy and because any other outcome would result in the same conclusion that current practice should not be modified to include the MusicGlove as a supplement to conventional home therapy [47]. To examine the time effect of therapy, we performed a repeated-measures analysis of variance (ANOVA) of absolute Box and Blocks scores from baseline through 1 mo posttherapy. Follow-up testing of the average changes compared with baseline across all subjects at each assessment was performed using one-tailed $t$-tests with Bonferroni corrections for multiple comparisons at the two time points (i.e., $\alpha=0.025$ ) [48].

Secondary end points were analyzed using a repeatedmeasures ANOVA of the absolute scores from baseline through 1 mo posttherapy. Follow-up tests were performed using two-tailed $t$-tests with Bonferroni corrections to test for significant changes within each group compared with baseline at both assessments (i.e., $\alpha=0.025$ ) and onetailed $t$-tests to test for significant differences between groups at 1 mo posttherapy ( $\alpha=0.05$ ).

We performed a post hoc analysis of the amount of use data recorded on the MusicGlove laptops, including the crossover data. First, we compared the total number of grips completed during MusicGlove therapy with a target dose of 2,700 total grips, based on a recommended dose of 300 repetitions/h suggested elsewhere [49], multiplied by 9 total hours of prescribed therapy. Then, to compare changes in amount of use as the study progressed, we performed two linear regression analyses for each subject using the total cumulative number of grips completed as the dependent variable and the day of the study as the independent variable for days 1 through 7 (the first week of therapy) and days 8 through 21 (the next two weeks of therapy) for each regression. Here we defined the boundary for a "day" as the grips completed between midnight on one calendar day and midnight on the next. The slopes of the resulting models provided estimates of the number of grips each subject completed per day during the first week of therapy and the next two weeks of therapy. We tested for a significant change in grips completed per day between these two periods across all subjects using a paired, one-tailed $t$-test.

We also analyzed every participant's performance in the MusicGlove game throughout the study (again including the crossover data), measured as the percentage of grips completed successfully out of the total number of grips requested during therapy. We compared each subject's average percentage on the first day of therapy with his or her percentage on the second day to test for a familiarization effect. We then compared each subject's average percentage on the second day of therapy with his or her percentage on the last day of the study to test for long-term improvements. In order to better understand why some participants did not comply with the prescribed regimen, we used a $t$-test to compare the change in percentage from day 1 to day 2 between the participants who did not meet the target dose of 2,700 total grips and those who did.

Finally, we performed an exploratory analysis of the relationship between the number of grips completed with the MusicGlove and the changes in the outcome measures. We did not include the crossover data in this analysis to eliminate any confounding effect of performing conventional therapy before MusicGlove therapy. For each outcome measure, we performed a linear regression 
analysis with the change in score compared with baseline at 1 mo posttherapy as the dependent variable and the number of grips completed as the independent variable.

\section{RESULTS}

Twenty three volunteers were assessed for eligibility in this study (see Consolidated Standards of Reporting Trials diagram, Figure 2). Of these, five subjects did not return for the second baseline assessment for unrelated reasons. One subject was excluded from analyses because of absence of follow-up data since the participant did not return for follow-up assessments.
Baseline characteristics for the remaining 17 subjects are presented in Table $\mathbf{1}$ and indicate no significant differences between the two treatment groups for any demographic or baseline measures except the Geriatric Depression Scale, which showed that the participants in the MusicGlove group had significantly greater levels of selfperceived depression at study start $(p=0.03$, Table 1$)$, though average scores in both groups fell short of the cutoff used to indicate major depression. One participant in the MusicGlove group had a Geriatric Depression Scale score of 11, indicating severe depression. Two of the participants in the conventional therapy group had a history of prior stroke, compared with none in the MusicGlove group.

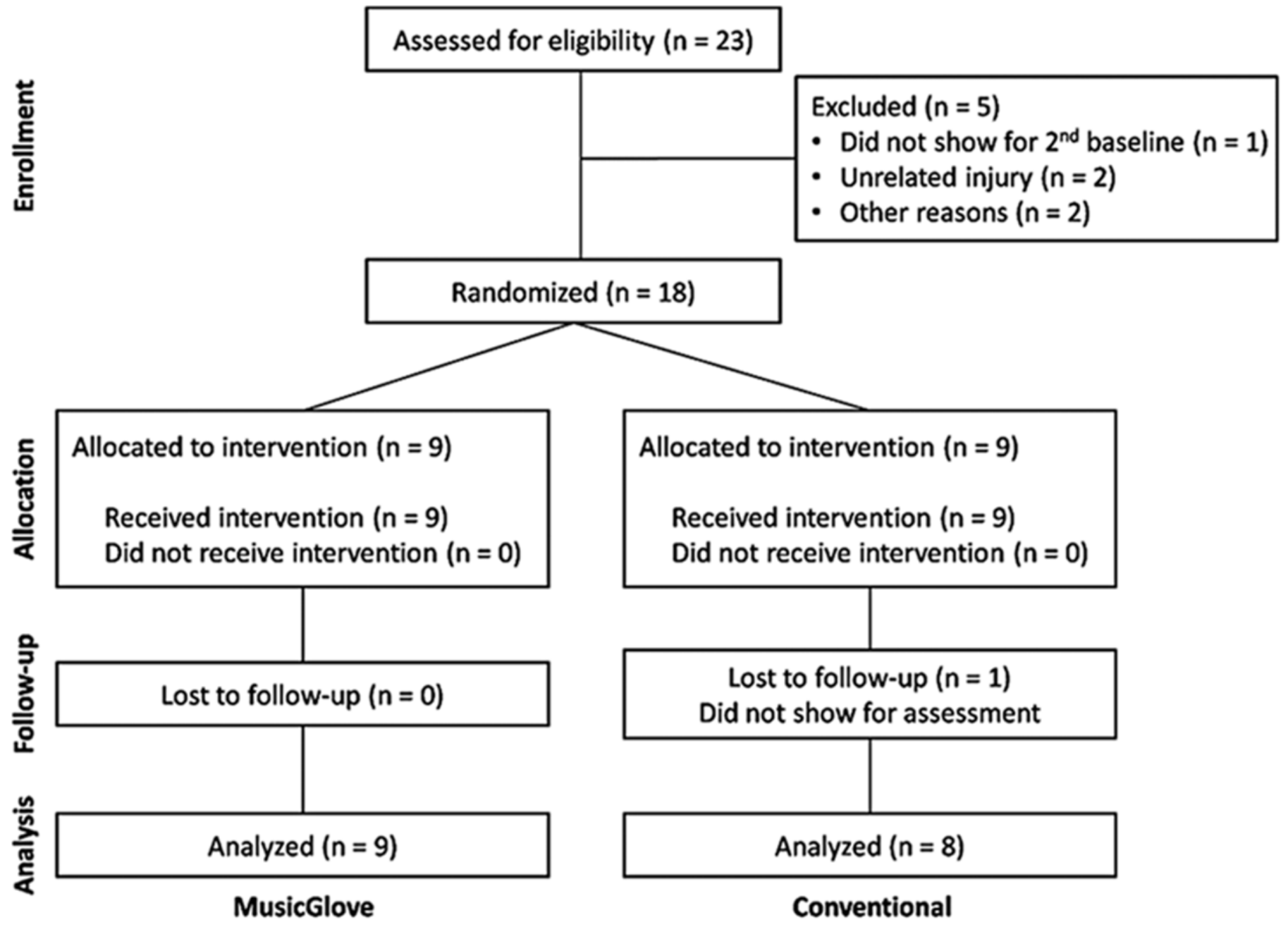

Figure 2.

Consolidated Standards of Reporting Trials flow diagram for study. 
No adverse events or safety issues occurred during the course of the study, and the participants reported no increase in pain after either training protocol. Participants in both groups typically required one follow-up call from the research therapist to solve technical issues or clarify the exercise regimen. Both groups reported close adherence to their respective training programs during the 3 wk exercise period, but some participants in the conventional therapy group reported persisting in their exercises after the exercise period ended, against the instructions of the research therapist.

For the Box and Blocks score, a significant difference was found between the two baseline assessments for all study participants, indicating a familiarization effect (increase of $2.2 \pm 3.1$ blocks, $p=0.009$ ). Thus, the change in Box and Blocks score was calculated from the second baseline assessment. No significant differences were found between the two baseline assessments for all secondary end points.

Analysis of the study's primary end point did not indicate a significant difference between groups at $1 \mathrm{mo}$ posttherapy, with the MusicGlove group improving by $2.3 \pm 6.2$ blocks and the conventional therapy group improving by $4.3 \pm 5.0$ blocks (Table 2). Repeatedmeasures ANOVA revealed that the main effect of time was significant on change in Box and Blocks score $(F(2,30)=3.85, p=0.03)$, but the group-time interaction effect was not significant $(p=0.23)$. Follow-up analysis revealed a significant change compared with baseline of $3.2 \pm 5.6$ blocks averaged across all subjects at $1 \mathrm{mo}$ posttherapy (one-tailed $t$-test, $p=0.02$; Figure 3 ).

Repeated-measures ANOVA of the secondary end points revealed that the main effect of time was significant on change in MAL QOM score $(F(2,30)=6.98, p=0.003)$ and MAL AOU score $(F(2,30)=9.45, p<0.001)$. There was also a significant group-time interaction effect on change in MAL QOM score $(F(2,30)=3.96, p=0.03)$ and a marginally significant group-time interaction effect on change in MAL AOU score $(F(2,30)=2.44, p=0.10)$. Repeated-measures ANOVA did not reveal any significant effects of treatment on Nine Hole Peg test or ARAT scores.

Follow-up testing revealed participants in the MusicGlove group had significant changes compared with baseline in MAL QOM and AOU scores both immediately posttherapy (two-tailed $t$-test, $p=0.03$ and $p=0.01$,

Table 1.

Participant demographics and baseline measures.

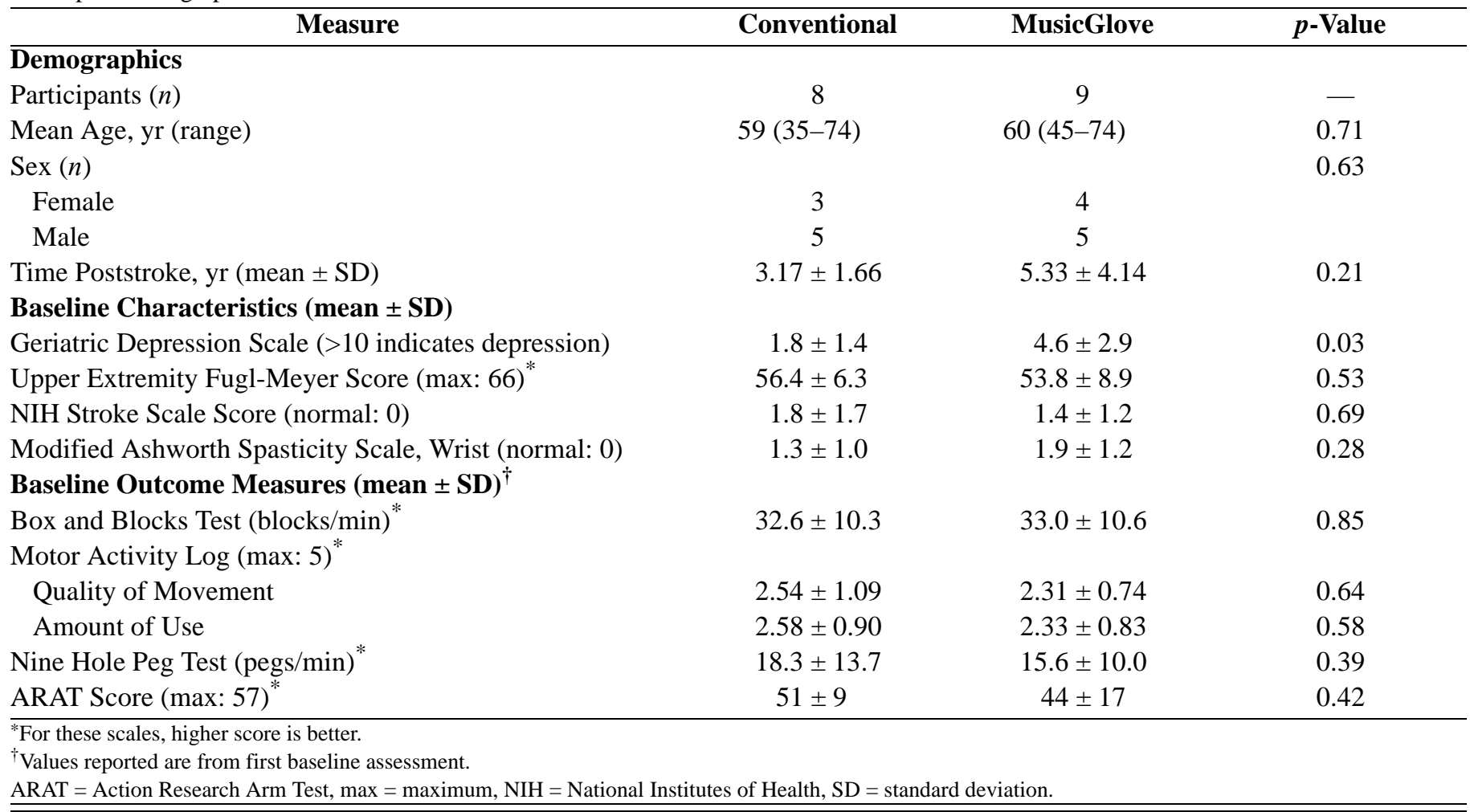


Table 2.

Change in outcome measures compared with baseline at 1 mo posttreatment. Unless otherwise indicated, values are given as mean \pm standard deviation.

\begin{tabular}{|c|c|c|c|c|}
\hline Outcome & $\begin{array}{l}\text { MusicGlove } \\
\quad(n=9)\end{array}$ & $\begin{array}{l}\text { Conventional } \\
\quad(n=8)\end{array}$ & $\begin{array}{c}\text { Mean Difference } \\
(95 \% \mathrm{CI})\end{array}$ & $\begin{array}{c}p \text {-Value } \\
\text { (one-tailed) }\end{array}$ \\
\hline Box and Blocks Test & $2.3 \pm 6.2$ & $4.3 \pm 5.0$ & $-1.9(-6.7$ to 2.9$)$ & 0.25 \\
\hline \multicolumn{5}{|l|}{ Motor Activity Log } \\
\hline Quality of Movement & $0.82 \pm 0.48^{*}$ & $0.09 \pm 0.58$ & $0.72(0.27$ to 1.17$)$ & 0.007 \\
\hline Amount of Use & $0.86 \pm 0.64^{*}$ & $0.26 \pm 0.69$ & $0.60(0.04$ to 1.16$)$ & 0.04 \\
\hline Nine Hole Peg Test & $1.3 \pm 6.9$ & $-0.2 \pm 6.0$ & $1.5(-4.1$ to 7.1$)$ & 0.33 \\
\hline
\end{tabular}

respectively) and at 1 mo posttherapy (two-tailed $t$-test, $p<0.001$ and $p=0.004$, respectively; see Figure 3). No significant changes compared with baseline were found for either outcome measure after conventional therapy. Subjects in the MusicGlove group had significantly greater improvements in both MAL QOM score and AOU score than subjects in the conventional therapy group at 1 mo posttherapy (mean differences of 0.72 and 0.60 points, one-tailed $t$-test, $p=0.007$ and $p=0.04$, respectively; Table 2 ).

Subjects were asked to fill out an activity log to record the amount of time they spent exercising during the 3 wk training period. Seven of the nine participants from the MusicGlove group and four of the eight participants from the control group filled out the activity log as requested. Participants in the MusicGlove group reported exercising for an average of $1.9 \mathrm{~h}$ total more than the control group during the training phase (10 h vs $8.1 \mathrm{~h}$, respectively), but this difference was not significant.

The total number of grips completed with the MusicGlove during the exercise period was recorded for each participant on the laptops provided. Data were not recovered for two of the participants due to technical errors. Of the remaining 15 participants (8 from the original MusicGlove group, 7 from the crossover group), 11 completed the target dose of 2,700 grips. Four individuals completed over 10,000 grips, with two of these completing over 30,000 grips (Figure 4(a)).

When we examined the time course of practice, we found that the cumulative number of grips completed each day was well represented by two lines, with an average $R^{2}$ value for a line fit across all subjects of $0.82 \pm$ 0.14 during the first week of therapy and an average $R^{2}$ of $0.86 \pm 0.16$ for a line fit to the data during the next two weeks. Using the slopes from these linear models, we estimated that the participants completed an average of $213 \pm 301$ grips per day during the first week of therapy and $466 \pm 641$ grips per day during the next two weeks of therapy, a significant difference ( $p=0.04$; Figure 4(b)). That is, the participants intensified their use of the MusicGlove after the first week of exposure.

We also examined the participants' performance in the MusicGlove game throughout the study, measured as the percentage of notes hit correctly. Performance increased throughout the study, with a significant increase of $11 \pm$ 13 percent from day 1 to day 2 of therapy ( $t$-test, $p=0.01$ ) and a nonsignificant increase from day 2 to the end of the study of $6 \pm 15$ percent.

Of the four participants who did not complete the target dose of 2,700 grips, three had an average performance of 15 percent or less (i.e., for every 100 notes presented, they successfully completed only 15 or less). Of the 11 participants who completed at least 2,700 grips, all 11 had an average performance of 22 percent or more, with a group mean of $53 \pm 15$ percent. Also, the group that completed at least 2,700 grips during therapy ( $n=$ 11) had an increase in performance of $14 \pm 14$ percent from day 1 to day 2 of therapy, while the group that did not complete the target dose $(n=4)$ had a decrease in performance of $1 \pm 2$ percent over the same time span, a significant difference ( $p=0.008)$.

In an exploratory analysis $(n=8)$, we found a significant linear relationship between the number of grips completed with the MusicGlove and the change in MAL AOU score at 1 mo posttherapy, with a slope of 0.05 points for every 1,000 grips completed with the MusicGlove $\left(R^{2}=0.61, p=0.02\right)$. No other outcome measures were significantly related to number of grips completed. 

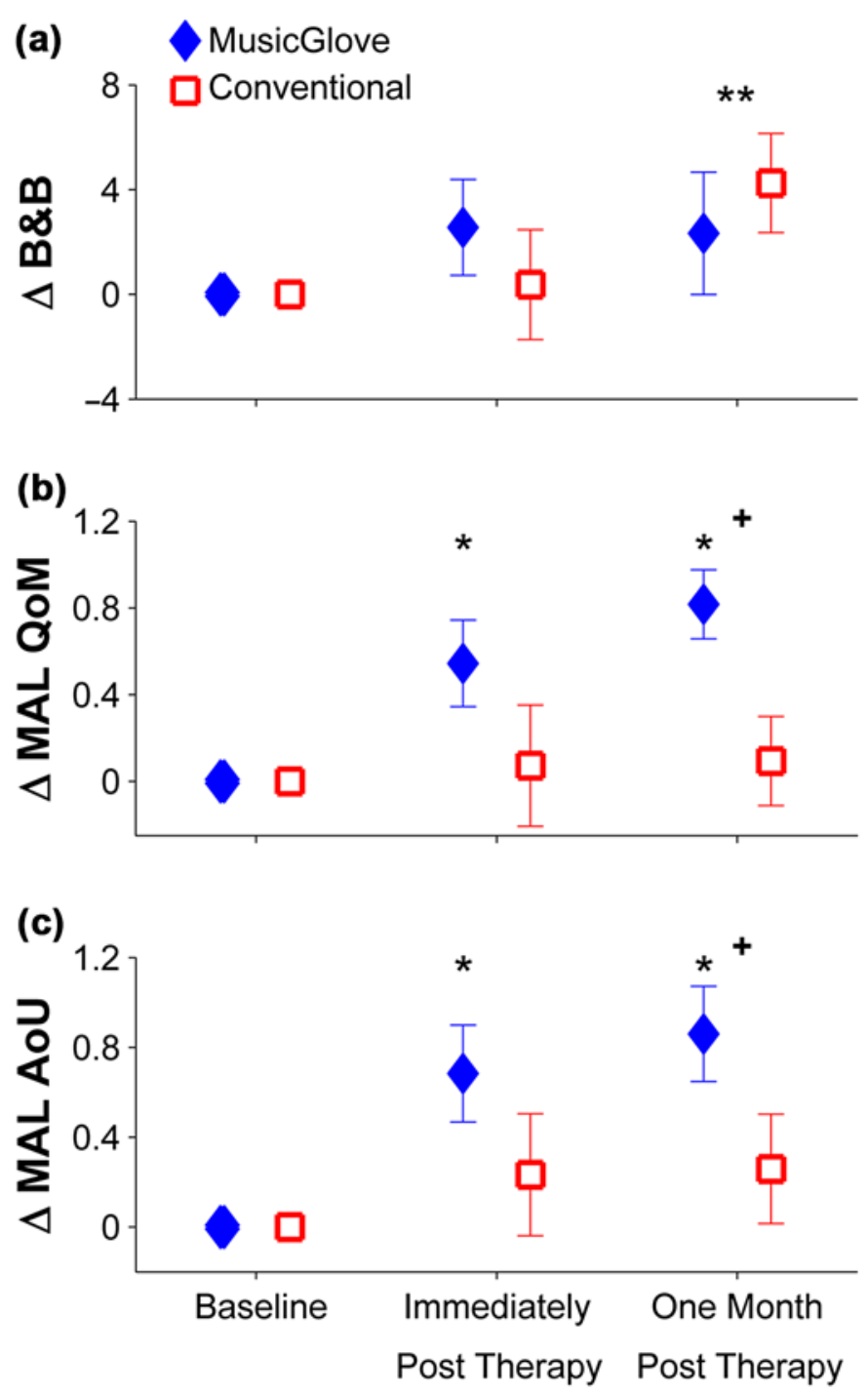

Figure 3.

(a) Longitudinal changes in Box and Blocks test (B\&B), (b) Motor Activity Log (MAL) Quality of Movement (QoM), and (c) MAL Amount of Use (AoU) throughout experiment. MusicGlove group (solid blue diamonds) had significantly greater improvements than conventional therapy group (open red squares) in both MAL scores 1 mo after therapy. Error bars indicate \pm 1 standard error. *Significant within-group changes compared with baseline (two-tailed $t$-test, $p<0.03$ ). ${ }^{* *}$ Significant changes across both groups compared with baseline (one-tailed $t$-test, $p<0.03)$. ${ }^{+}$Significant differences between groups at 1 mo posttherapy (one-tailed $t$-test, $p<0.05$ ).

Finally, we performed two more exploratory analyses. First, we noticed that one of the MusicGlove participants had a decrease in Box and Blocks score from baseline of
10 blocks at 1 mo posttherapy and a decrease of only 2 blocks immediately after therapy. This was a large change relative to all other participants (greater than two standard deviations away from the group mean), and thus we speculated that this decrease may have been due to poor motivation on that particular day. When we removed this participant from the analysis, the change in Box and Blocks score compared with baseline for the MusicGlove group increased to $3.9 \pm 1.7$ blocks at 1 mo posttherapy. Second, for all end points, we also analyzed the combined data from the MusicGlove group and the data from the crossover period for the conventional therapy group, in which they also received MusicGlove therapy (resulting in an $n=17$ for the MusicGlove group). Here, the changes in outcome measures for the crossover group were calculated from the "baseline" of the 1 mo posttherapy assessment after conventional therapy. In this analysis, the MusicGlove group had a significantly greater change in ARAT score than the conventional therapy group $(n=8)$ at 1 mo posttherapy (mean difference of 2.4 points, onetailed $t$-test, $p=0.046$ ). Thus, both of these exploratory analyses supported positive effects of MusicGlove training, although the first relied on an ad hoc outlier removal and the second combined change scores without a prior history of participation in conventional training with change scores that were dependent on a prior history of participation in conventional training.

\section{DISCUSSION}

\section{Increased Functional Use of Hand After MusicGlove Therapy}

Notably, some of the participants in the conventional therapy group did not adhere to the study protocol and continued exercising after the exercise period. This may explain why the conventional therapy group had a substantial increase in Box and Blocks score at 1 mo posttherapy after a relatively small increase immediately posttherapy (Figure 3). Yet, despite the fact that there was a significant increase in Box and Blocks score across both the MusicGlove and conventional therapy groups at 1 mo posttherapy, only the MusicGlove group converted this improvement in gripping function into increased self-reported functional use of the hand as measured by the MAL scores.

One possible explanation for this increased functional use of the hand after MusicGlove therapy is that thumb 
(a)

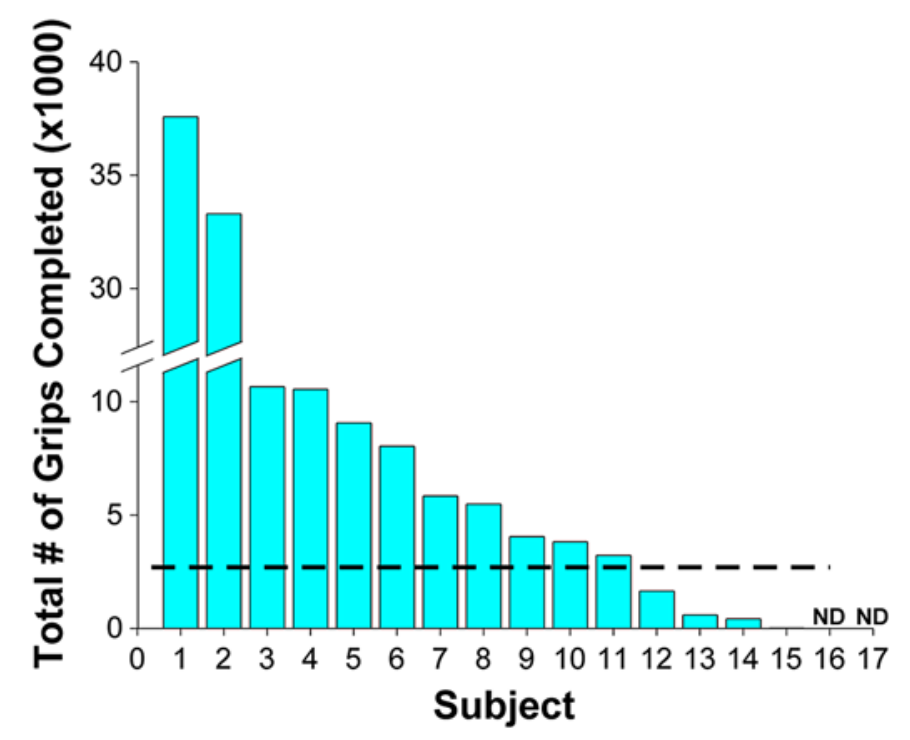

(b)

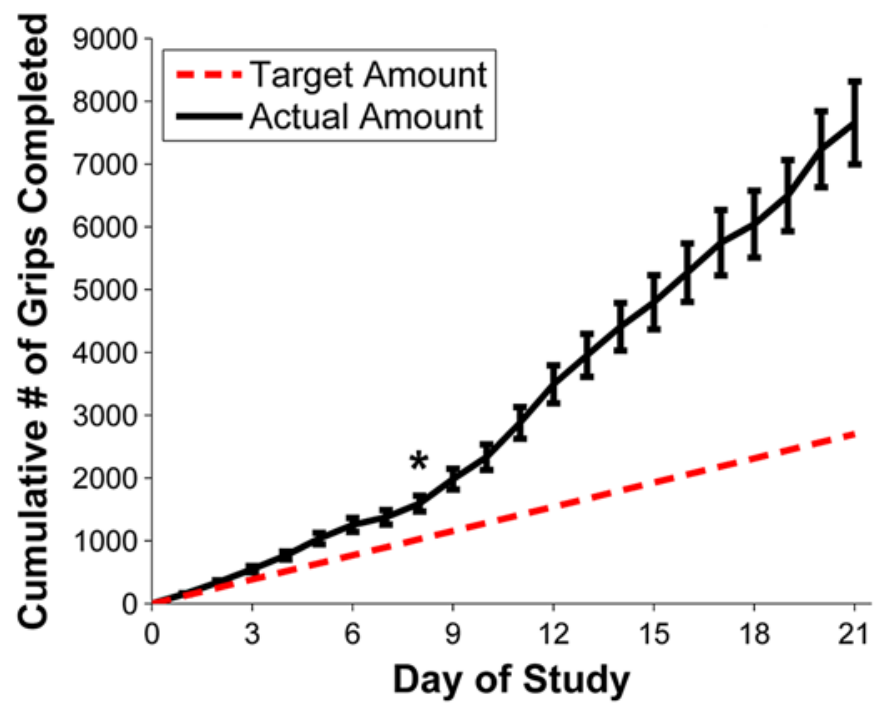

Figure 4.

(a) Number of grips completed with MusicGlove by each study participant, including crossover data. Note break in $y$-axis from 12,000 to 27,000 grips, which was inserted for readability. Dashed line represents target dose of 2,700 total grips. (b) Mean cumulative number of grips completed as function of day in study for all study participants, including crossover data. Dashed line represents expected progression of cumulative number of grips if subjects practiced 300 repetitions $/ \mathrm{h}, 3 \mathrm{~h} / \mathrm{wk}$, for $3 \mathrm{wk}$. Average number of grips completed per day across all subjects increased significantly from first week of therapy to next 2 wk of therapy $(p=$ 0.04). ${ }^{*}$ Transition point (i.e., day 8). Error bars show \pm 1 standard error. ND = data not available for participants. opposition is critical for functional use of the hand, and the MusicGlove is more effective at promoting intensive training of thumb opposition than conventional therapy. Another more general possibility is that functional use of the hand requires several fundamental movement patterns of the fingers and thumb and that the MusicGlove is effective at training these patterns. Indeed, a study that used principal component decomposition to analyze the movement patterns associated with functional use of the hand found that $>90$ percent of the variance in hand kinematics could be explained by nine finger-thumb movement patterns similar to ones trained by the MusicGlove [50]. In this framework, practicing a key movement component that is used in a range of daily tasks may be more efficient at inducing functional recovery than practicing the individual tasks themselves [51-53], a hypothesis one could call "componentspecific training" in order to contrast it with the widely advocated approach of "task-specific training." A third possibility is that practice with the MusicGlove simply made the participants pay more attention to their hand, and thus they were more inclined to report an increased use of it in daily tasks.

\section{Comparison with Previous Hand Therapy Studies}

Our previous clinic-based study of the MusicGlove [27-28] included participants with a chronic stroke of similar duration and similar levels of initial hand impairment as the current study. However, in that study, subjects trained for 2 wk in six $1 \mathrm{~h}$ sessions with the MusicGlove under continuous supervision. The change in Box and Blocks score was 3.2 blocks after 6 h of clinical therapy versus 2.3 blocks after $9 \mathrm{~h}$ of home-based therapy in the current study. Thus, the gains achievable at home were comparable to those achieved in the clinic, though there is a mild indication that clinic-based therapy is more efficient at improving gripping function.

The largest trial of hand therapy reported to date is the EXCITE trial, which used large amounts of graded, task-oriented exercises [54-55]. Specifically, participants in the EXCITE trial received a total of $60 \mathrm{~h}$ of therapy for up to $6 \mathrm{~h} / \mathrm{d}, 5 \mathrm{~d} / \mathrm{wk}$, for $2 \mathrm{wk}$, during which they were prevented from using their nonimpaired limb. In the present study, the functional gains following MusicGlove use were 0.82 for MAL QOM and 0.86 for MAL AOU. Following the EXCITE trial, the functional gains were 1.12 for MAL QOM and 1.07 for MAL AOU. This finding that participants who used the MusicGlove achieved about 75 to 80 percent of the benefit reported on the 
MAL for the EXCITE trial despite less training time, absence of a constraint on their nonimpaired hand, and use of home therapy rather than therapist-supervised training is notable.

Comparing the outcomes of the present study with other home-based hand therapy trials is difficult because of variations in study protocol, amount of therapist guidance provided, and outcome measures [14,17-23]. However, the improvements in Box and Blocks score seen here are similar to the gains observed in a prior home-based telerehabilitation study with chronic stroke subjects that incorporated virtual line-tracking with a finger goniometer, which reported changes of 2.0 and 4.9 blocks in the two study groups [16]. That study included five video calls between the therapist and the patient over a $10 \mathrm{~d}$ training period, while the present study used less interaction.

\section{Influence of Motivating Factors on Dose of Exercise and Long-Term Outcomes}

There is growing consensus that individuals typically perform far too few exercise repetitions to maximize recovery after stroke $[7,49,56]$. Indeed, our exploratory analysis of the relationship between number of grips completed and outcomes suggested that, at least in terms of increasing self-reported functional use of the hand, there is a positive correlation between the dose of therapy and outcomes. However, the slope of that correlation was small, indicating that thousands of additional exercise repetitions are required in order to promote increased functional use.

As mentioned in the "Methods" section, a recent study suggested 300 repetitions in a $1 \mathrm{~h}$ therapy session was an appropriate target [49]; this is an order of magnitude more than the number of repetitions typically performed in guided therapy sessions [7]. Of the 15 participants in the present study whose MusicGlove usage data were collected successfully, 11 exceeded this target. Of the four participants who did not, three had very low levels of performance in the MusicGlove game, in terms of percentage of notes successfully hit, suggesting that the therapy was difficult for them. Further, none of these four participants improved their performance from day 1 to day 2 of therapy, which could have reduced their motivation to continue. This is in line with other studies that have shown high levels of difficulty or lack of improvement in a task can reduce motivation to persist [57-59]. Based on the results of this study, a minimum average performance of 20 percent in the MusicGlove game may be an important factor for maintaining motivation during home therapy with the device.

Interestingly, instead of observing a novelty effect in which the participants initially used the MusicGlove more frequently and then tapered off their use, we instead observed that the participants significantly increased their use of the device after the first week of therapy, completing on average over 200 additional grips per day during the next 2 wk. Some of this increase may have been due to increased performance in the game (i.e., the participants completed a higher percentage of the grips that were requested during a given song, resulting in more grips per day). However, performance data from the laptops showed an average improvement of only 6 percent from the second to the final day of therapy, which would have resulted in an increase of only about 30 additional grips per day if the amount of practice time had remained the same. Thus, a better explanation of the increase in grips completed per day is that the participants became more interested and motivated to use the MusicGlove after their initial exposure to it.

Indeed, the use of motivating factors such as music and video games during therapy may be an important approach to improve the long-term outcomes of a rehabilitation program. Music has been shown to encourage movement via tightly coupled interaction between the auditory and motor cortices, which may improve motor recovery [26,60-61]. Also, the use of music during therapy may enhance neural reorganization, thus increasing functional outcomes from the therapy [25,62-69]. The use of video games not only creates an immersive exercise experience but can also encourage a high number of repetitions, create an appropriate level of challenge, reward progress, and provide feedback on improvements over time. The MusicGlove includes each of these features, all of which have been linked to improved longterm outcomes of therapy [58,70-73] and likely contributed to the significant improvements in motor function after MusicGlove therapy in the present study.

Of particular note is that the participants in the MusicGlove group had significantly higher levels of selfreported depression on the Geriatric Depression Scale than the conventional therapy group at baseline (i.e., they were more likely to respond negatively to questions pertaining to their quality of life). This may have been expected to limit functional improvements in this group since depressive symptoms have been shown to reduce outcomes from rehabilitation after stroke [74] and are 
correlated with an inability to carry out many activities of daily living [75]. Indeed, one participant did report poor adherence to the study protocol because of severe depression. However, for the rest of the participants, this was not the case. We did not reassess the participants' scores on the Geriatric Depression Scale at the 1 mo follow-up, but future studies should assess whether exercising with the MusicGlove can improve psychological well-being. This may in turn promote increased functional use of the hand, such as we observed here.

\section{Limitations and Future Directions}

Limitations of this study include a small sample size, the conventional therapy group's poor adherence to the study protocol by continuing to exercise after the $3 \mathrm{wk}$ training period, the lack of an accurate measure of the number of exercise repetitions the participants in the conventional therapy group performed during the experiment, subject dropout, and incomplete data collection on two of the MusicGlove laptops because of technical errors. Common technical difficulties with the MusicGlove included an inability to double-click the desktop icon that opened the MusicGlove application on the laptop, difficulties using a tracking pad to control the laptop cursor, difficulty properly exiting the MusicGlove application, and difficulty turning the computer on and off. There were few technical issues with the MusicGlove hardware itself.

Future research should explore the use of MusicGlove therapy in individuals with subacute stroke and other populations that exhibit hand impairment, such as those with traumatic brain injury and spinal cord injury. Other studies could also examine which motivating factors (e.g., music or video games) are most effective for facilitating large amounts of movement practice and improving long-term functional outcomes and the mechanisms by which these factors influence self-report of functional use of the hand. Finally, the ability of the MusicGlove to accurately record the number of movement repetitions performed during exercise makes it well-suited for use in a study that explores the relationship between dose and therapeutic outcomes. Further studies could expand on the small exploratory analysis we presented here by examining this relationship across a larger sample size and under a more closely controlled therapeutic regimen.

\section{CONCLUSIONS}

The results of this study confirmed that the MusicGlove is viable and effective for home therapy, and it motivated users to complete a high number of therapeutic gripping movements. We did not observe a significant difference between MusicGlove therapy and conventional therapy in the primary end point, but we did observe a significant difference in favor of the MusicGlove in two of the secondary end points (MAL AOU and QOM).

\section{ACKNOWLEDGMENTS}

\section{Author Contributions:}

Study concept and design: N. Friedman, D. J. Reinkensmeyer, S. C. Cramer, D. K. Zondervan.

Acquisition of data: R. Augsburger, E. Chang.

Analysis and interpretation of data: D. K. Zondervan, N. Friedman, D. J. Reinkensmeyer, S. C. Cramer.

Drafting of manuscript: D. K. Zondervan, N. Friedman,

D. J. Reinkensmeyer, S. C. Cramer.

Statistical analysis: D. K. Zondervan, N. Friedman, D. J. Reinkensmeyer, S. C. Cramer.

Obtained funding: D. K. Zondervan, N. Friedman, D. J. Reinkensmeyer. Administrative, technical, or material support: E. Chang, X. Zhao.

Study supervision: N. Friedman, D. J. Reinkensmeyer, R. Augsburger.

Financial Disclosures: Daniel K. Zondervan, Nizan Friedman, and David J. Reinkensmeyer have a financial interest in Flint Rehabilitation Devices, LLC, a company that develops and sells rehabilitation devices, including MusicGlove. Steven C. Cramer serves as a consultant for GlaxoSmithKline, RAND Corporation, Dart Neuroscience, and MicroTransponder, and is a cofounder of personalRN. He acknowledges support from K24 HD074722 and UL1 TR000153. The terms of these arrangements have been reviewed and approved by the University of California, Irvine, in accordance with its conflict of interest policies. The remaining authors declare that they have no competing interests.

Funding/Support: This material was based on work supported by the National Institutes of Health (grant 1R43HD074331-01) and the Department of Education (National Institute on Disability and Rehabilitation Research grant H133S120032).

Additional Contributions: Thank you to Derek Yano for assistance with data entry. The project described was supported by the National Center for Research and the National Center for Advancing Translational Sciences, National Institutes of Health, through Grant UL1TR000153. The content is solely the responsibility of the authors and does not necessarily represent the official views of the National Institutes of Health.

Institutional Review: The study protocol was approved by the University of California at Irvine's Institutional Review Board.

Participant Follow-up: The authors plan to inform participants of the publication of this study. 


\section{REFERENCES}

1. Dobkin BH. Neurologic rehabilitation. Philadelphia (PA): F. A. Davis; 1996.

2. Sawaki L. Use-dependent plasticity of the human motor cortex in health and disease. IEEE Eng Med Biol Mag. 2005;24(1):36-39. [PMID:15709534] http://dx.doi.org/10.1109/MEMB.2005.1384098

3. Ada L, Dorsch S, Canning CG. Strengthening interventions increase strength and improve activity after stroke: A systematic review. Aust J Physiother. 2006;52(4):241-48. [PMID:17132118] http://dx.doi.org/10.1016/S0004-9514(06)70003-4

4. van der Lee JH, Snels IA, Beckerman H, Lankhorst GJ, Wagenaar RC, Bouter LM. Exercise therapy for arm function in stroke patients: A systematic review of randomized controlled trials. Clin Rehabil. 2001;15(1):20-31. [PMID:11237158] http://dx.doi.org/10.1191/026921501677557755

5. Kloosterman MG, Snoek GJ, Jannink MJ. Systematic review of the effects of exercise therapy on the upper extremity of patients with spinal-cord injury. Spinal Cord. 2009;47(3):196-203. [PMID:18825160] http://dx.doi.org/10.1038/sc.2008.113

6. Nudo RJ. Postinfarct cortical plasticity and behavioral recovery. Stroke. 2007;38(2 Suppl):840-45. [PMID:17261749] http://dx.doi.org/10.1161/01.STR.0000247943.12887.d2

7. Lang CE, Macdonald JR, Reisman DS, Boyd L, Jacobson Kimberley T, Schindler-Ivens SM, Hornby TG, Ross SA, Scheets PL. Observation of amounts of movement practice provided during stroke rehabilitation. Arch Phys Med Rehabil. 2009;90(10):1692-98. [PMID:19801058] http://dx.doi.org/10.1016/j.apmr.2009.04.005

8. Byl NN, Pitsch EA, Abrams GM. Functional outcomes can vary by dose: Learning-based sensorimotor training for patients stable poststroke. Neurorehabil Neural Repair. 2008;22(5):494-504. [PMID:18780884] http://dx.doi.org/10.1177/1545968308317431

9. Cox KL, Burke V, Gorely TJ, Beilin LJ, Puddey IB. Controlled comparison of retention and adherence in home- vs center-initiated exercise interventions in women ages 4065 years: The S.W.E.A.T. Study (Sedentary Women Exercise Adherence Trial). Prev Med. 2003;36(1):17-29.

[PMID:12473421]

http://dx.doi.org/10.1006/pmed.2002.1134

10. Rejeski WJ, Brawley LR, Ettinger W, Morgan T, Thompson C. Compliance to exercise therapy in older participants with knee osteoarthritis: Implications for treating disability. Med Sci Sports Exerc. 1997;29(8):977-85.

[PMID:9268953]

http://dx.doi.org/10.1097/00005768-199708000-00001
11. Sluijs EM, Kok GJ, van der Zee J. Correlates of exercise compliance in physical therapy. Phys Ther. 1993;73(11): 771-82, discussion 783-86. [PMID:8234458]

12. Turton A, Fraser C. The use of home therapy programmes for improving recovery of the upper limb following stroke. Br J Occup Ther. 1990;53(11):457-62. http://dx.doi.org/10.1177/030802269005301104

13. Jurkiewicz MT, Marzolini S, Oh P. Adherence to a homebased exercise program for individuals after stroke. Top Stroke Rehabil. 2011;18(3):277-84. [PMID:21642065] http://dx.doi.org/10.1310/tsr1803-277

14. Barzel A, Liepert J, Haevernick K, Eisele M, Ketels G, Rijntjes M, van den Bussche H. Comparison of two types of Constraint-Induced Movement Therapy in chronic stroke patients: A pilot study. Restor Neurol Neurosci. 2009;27(6):673-80. [PMID:20042791]

15. Barzel A, Ketels G, Stark A, Tetzlaff B, Daubman A, Wegscheider K, van den Bussche H, Scherer M. Home-based constraint-induced movement therapy for patients with upper limb dysfunction after stroke (HOMECIMT): A cluster-randomized, controlled trial. Lancet Neurol. 2015; 14(9):893-902. [PMID:26231624]

http://dx.doi.org/10.1016/S1474-4422(15)00147-7

16. Carey JR, Durfee WK, Bhatt E, Nagpal A, Weinstein SA, Anderson KM, Lewis SM. Comparison of finger tracking versus simple movement training via telerehabilitation to alter hand function and cortical reorganization after stroke. Neurorehabil Neural Repair. 2007;21(3):216-32.

[PMID:17351083] http://dx.doi.org/10.1177/1545968306292381

17. Laver KE, Schoene D, Crotty M, George S, Lannin NA, Sherrington C. Telerehabilitation services for stroke. Cochrane Database Syst Rev. 2013;12:CD010255. [PMID:24338496]

18. Hara Y, Ogawa S, Tsujiuchi K, Muraoka Y. A home-based rehabilitation program for the hemiplegic upper extremity by power-assisted functional electrical stimulation. Disabil Rehabil. 2008;30(4):296-304. [PMID:17852312] http://dx.doi.org/10.1080/09638280701265539

19. Donoso Brown EV, McCoy SW, Fechko AS, Price R, Gilbertson T, Moritz CT. Preliminary investigation of an electromyography-controlled video game as a home program for persons in the chronic phase of stroke recovery. Arch Phys Med Rehabil. 2014;95(8):1461-69. [PMID:24657112] http://dx.doi.org/10.1016/j.apmr.2014.02.025

20. King M, Hijmans JM, Sampson M, Satherley J, Hale L. Home-based stroke rehabilitation using computer gaming. N Z J Physiother. 2012;40(3):128-34.

21. Slijper A, Svensson KE, Backlund P, Engström H, Sunnerhagen KS. Computer game-based upper extremity training in the home environment in stroke persons: A single subject design. J Neuroeng Rehabil. 2014;11:35. 


\section{[PMID:24625289]}

http://dx.doi.org/10.1186/1743-0003-11-35

22. Villeneuve M, Lamontagne A. Playing piano can improve upper extremity function after stroke: case studies. Stroke Res Treat. 2013;2013:159105. [PMID:23533954] http://dx.doi.org/10.1155/2013/159105

23. Coupar F, Pollock A, Legg LA, Sackley C, van Vliet P. Home-based therapy programmes for upper limb functional recovery following stroke. Cochrane Database Syst Rev. 2012;5:CD006755. [PMID:22592715]

24. Hijmans JM, Hale LA, Satherley JA, McMillan NJ, King MJ. Bilateral upper-limb rehabilitation after stroke using a movement-based game controller. J Rehabil Res Dev. 2011;48(8):1005-13. [PMID:22068375] http://dx.doi.org/10.1682/JRRD.2010.06.0109

25. Safranek BR. The use of music therapy in stroke rehabilitation. Music Ther. 2011;1:1-13.

26. Yoo J. The role of therapeutic instrumental music performance in hemiparetic arm rehabilitation. Music Ther Perspect. 2009; 27(1):16-24. http://dx.doi.org/10.1093/mtp/27.1.16

27. Friedman N, Chan V, Reinkensmeyer AN, Beroukhim A, Zambrano GJ, Bachman M, Reinkensmeyer DJ. Retraining and assessing hand movement after stroke using the MusicGlove: Comparison with conventional hand therapy and isometric grip training. J Neuroeng Rehabil. 2014;11:76. [PMID:24885076] http://dx.doi.org/10.1186/1743-0003-11-76

28. Friedman N, Chan V, Zondervan D, Bachman M, Reinkensmeyer DJ. MusicGlove: motivating and quantifying hand movement rehabilitation by using functional grips to play music. Conf Proc IEEE Eng Med Biol Soc. 2011; 2011:2359-63. [PMID:22254815] http://dx.doi.org/10.1109/IEMBS.2011.6090659

29. Mathiowetz V, Volland G, Kashman N, Weber K. Adult norms for the Box and Block Test of manual dexterity. Am J Occup Ther. 1985;39(6):386-91. [PMID:3160243] http://dx.doi.org/10.5014/ajot.39.6.386

30. Platz T, Pinkowski C, van Wijck F, Kim I-H, di Bella P, Johnson G. Reliability and validity of arm function assessment with standardized guidelines for the Fugl-Meyer Test, Action Research Arm Test and Box and Block Test: A multicentre study. Clin Rehabil. 2005;19(4):404-11. [PMID:15929509] http://dx.doi.org/10.1191/0269215505cr832oa

31. Uswatte G, Taub E, Morris D, Vignolo M, McCulloch K. Reliability and validity of the upper-extremity Motor Activity Log-14 for measuring real-world arm use. Stroke. 2005;36(11):2493-96. [PMID:16224078] http://dx.doi.org/10.1161/01.STR.0000185928.90848.2e

32. Uswatte G, Taub E, Morris D, Light K, Thompson PA. The Motor Activity Log-28: Assessing daily use of the hemiparetic arm after stroke. Neurology. 2006;67(7):1189-94.

\section{[PMID:17030751]}

http://dx.doi.org/10.1212/01.wnl.0000238164.90657.c2

33. Oxford Grice K, Vogel KA, Le V, Mitchell A, Muniz S, Vollmer MA. Adult norms for a commercially available Nine Hole Peg Test for finger dexterity. Am J Occup Ther. 2003;57(5):570-73. [PMID:14527120] http://dx.doi.org/10.5014/ajot.57.5.570

34. Yozbatiran N, Der-Yeghiaian L, Cramer SC. A standardized approach to performing the action research arm test. Neurorehabil Neural Repair. 2008;22(1):78-90.

[PMID:17704352] http://dx.doi.org/10.1177/1545968307305353

35. Yesavage JA, Brink TL, Rose TL, Lum O, Huang V, Adey M, Leirer VO; Yesavage. Development and validation of a geriatric depression screening scale: A preliminary report. J Psychiatr Res. 1982-1983;17(1):37-49. [PMID:7183759] http://dx.doi.org/10.1016/0022-3956(82)90033-4

36. Gladstone DJ, Danells CJ, Black SE. The Fugl-Meyer assessment of motor recovery after stroke: A critical review of its measurement properties. Neurorehabil Neural Repair. 2002;16(3):232-40. [PMID:12234086]

http://dx.doi.org/10.1177/154596802401105171

37. Lyden P, Brott T, Tilley B, Welch KM, Mascha EJ, Levine S, Haley EC, Grotta J, Marler J; NINDS TPA Stroke Study Group. Improved reliability of the NIH Stroke Scale using video training. Stroke. 1994;25(11):2220-26.

[PMID:7974549]

http://dx.doi.org/10.1161/01.STR.25.11.2220

38. Bohannon RW, Smith MB. Interrater reliability of a modified Ashworth scale of muscle spasticity. Phys Ther. 1987; 67(2):206-7. [PMID:3809245]

39. Hummel F, Celnik P, Giraux P, Floel A, Wu W-H, Gerloff C, Cohen LG. Effects of non-invasive cortical stimulation on skilled motor function in chronic stroke. Brain. 2005; 128(Pt 3):490-99. [PMID:15634731] http://dx.doi.org/10.1093/brain/awh369

40. Chen H-M, Chen CC, Hsueh I-P, Huang S-L, Hsieh C-L. Test-retest reproducibility and smallest real difference of 5 hand function tests in patients with stroke. Neurorehabil Neural Repair. 2009;23(5):435-40. [PMID:19261767] http://dx.doi.org/10.1177/1545968308331146

41. Meinert CL, Tonascia S. Clinical trials: Design, conduct, and analysis. New York (NY): Oxford University Press; 1986.

42. Housman SJ, Scott KM, Reinkensmeyer DJ. A randomized controlled trial of gravity-supported, computer-enhanced arm exercise for individuals with severe hemiparesis. Neurorehabil Neural Repair. 2009;23(5):505-14.

[PMID:19237734] http://dx.doi.org/10.1177/1545968308331148

43. Takahashi CD, Der-Yeghiaian L, Le V, Motiwala RR, Cramer SC. Robot-based hand motor therapy after stroke. 
Brain. 2008 Feb;131(Pt 2):425-437. PMID:18156154

http://dx.doi.org/10.1093/brain/awm311

44. Feys HM, De Weerdt WJ, Selz BE, Cox Steck GA, Spichiger R, Vereeck LE, Putman KD, Van Hoydonck GA. Effect of a therapeutic intervention for the hemiplegic upper limb in the acute phase after stroke: A single-blind, randomized, controlled multicenter trial. Stroke. 1998; 29(4):785-92. [PMID:9550512]

http://dx.doi.org/10.1161/01.STR.29.4.785

45. Kwakkel G, Wagenaar RC, Twisk JW, Lankhorst GJ, Koetsier JC. Intensity of leg and arm training after primary middlecerebral-artery stroke: A randomised trial. Lancet. 1999; 354(9174):191-96. [PMID:10421300] http://dx.doi.org/10.1016/S0140-6736(98)09477-X

46. Brunner IC, Skouen JS, Strand LI. Recovery of upper extremity motor function post stroke with regard to eligibility for constraint-induced movement therapy. Top Stroke Rehabil. 2011;18(3):248-57. [PMID:21642062] http://dx.doi.org/10.1310/tsr1803-248

47. Ruxton GD, Neuhäuser M. When should we use one-tailed hypothesis testing? Methods Ecol Evol. 2010;1(2):114-17. http://dx.doi.org/10.1111/j.2041-210X.2010.00014.x

48. Holm S. A simple sequentially rejective multiple test procedure. Scand J Stat. 1979;6(2):65-70.

49. Birkenmeier RL, Prager EM, Lang CE. Translating animal doses of task-specific training to people with chronic stroke in 1-hour therapy sessions: A proof-of-concept study. Neurorehabil Neural Repair. 2010;24(7):620-35.

[PMID:20424192]

http://dx.doi.org/10.1177/1545968310361957

50. Thakur PH, Bastian AJ, Hsiao SS. Multidigit movement synergies of the human hand in an unconstrained haptic exploration task. J Neurosci. 2008;28(6):1271-81.

[PMID:18256247]

http://dx.doi.org/10.1523/JNEUROSCI.4512-07.2008

51. Klein J, Spencer SJ, Reinkensmeyer DJ. Breaking it down is better: Haptic decomposition of complex movements aids in robot-assisted motor learning. IEEE Trans Neural Syst Rehabil Eng. 2012;20(3):268-75. [PMID:22531825] http://dx.doi.org/10.1109/TNSRE.2012.2195202

52. Milot M-H, Spencer SJ, Chan V, Allington JP, Klein J, Chou C, Bobrow JE, Cramer SC, Reinkensmeyer DJ. A crossover pilot study evaluating the functional outcomes of two different types of robotic movement training in chronic stroke survivors using the arm exoskeleton BONES. J Neuroeng Rehabil. 2013;10:112. [PMID:24354476]

http://dx.doi.org/10.1186/1743-0003-10-112

53. Zondervan DK, Augsburger R, Bodenhoefer B, Friedman N, Reinkensmeyer DJ, Cramer SC. Machine-based, selfguided home therapy for individuals with severe arm impairment after stroke: A randomized controlled trial. Neurorehabil Neural Repair. 2015;29(5):395-406.

\section{[PMID:25273359]}

http://dx.doi.org/10.1177/1545968314550368

54. Wolf SL, Winstein CJ, Miller JP, Thompson PA, Taub E, Uswatte G, Morris D, Blanton S, Nichols-Larsen D, Clark PC. Retention of upper limb function in stroke survivors who have received constraint-induced movement therapy: The EXCITE randomised trial. Lancet Neurol. 2008;7(1): 33-40. [PMID:18077218] http://dx.doi.org/10.1016/S1474-4422(07)70294-6

55. Cramer SC. The EXCITE trial: a major step forward for restorative therapies in stroke. Stroke. 2007;38(7):2204-5. [PMID:17540965]

56. Plautz EJ, Milliken GW, Nudo RJ. Effects of repetitive motor training on movement representations in adult squirrel monkeys: Role of use versus learning. Neurobiol Learn Mem. 2000;74(1):27-55. [PMID:10873519]

http://dx.doi.org/10.1006/nlme.1999.3934

57. Duarte JE, Gebrekristos B, Perez S, Rowe JB, Sharp K, Reinkensmeyer DJ. Effort, performance, and motivation: Insights from robot-assisted training of human golf putting and rat grip strength. IEEE Int Conf Rehabil Robot. 2013; 2013:6650461. [PMID:24187278] http://dx.doi.org/10.1109/ICORR.2013.6650461

58. Guadagnoli MA, Lee TD. Challenge point: A framework for conceptualizing the effects of various practice conditions in motor learning. J Mot Behav. 2004;36(2):212-24. [PMID:15130871] http://dx.doi.org/10.3200/JMBR.36.2.212-224

59. Reinkensmeyer DJ, Housman SJ. "If I can't do it once, why do it a hundred times?": Connecting volition to movement success in a virtual environment motivates people to exercise the arm after stroke. Proceedings of the Virtual Rehabilitation Conference; 2007 Sep 27-29; Venice, Italy.

60. Bangert M, Peschel T, Schlaug G, Rotte M, Drescher D, Hinrichs H, Heinze HJ, Altenmüller E. Shared networks for auditory and motor processing in professional pianists: Evidence from fMRI conjunction. Neuroimage. 2006; 30(3):917-26. [PMID:16380270] http://dx.doi.org/10.1016/j.neuroimage.2005.10.044

61. Fujioka T, Ween JE, Jamali S, Stuss DT, Ross B. Changes in neuromagnetic beta-band oscillation after music-supported stroke rehabilitation. Ann N Y Acad Sci. 2012;1252(1): 294-304. [PMID:22524371] http://dx.doi.org/10.1111/j.1749-6632.2011.06436.x

62. Gordon WA, Hibbard MR. Critical issues in cognitive remediation. Neuropsychology. 1992;6(4):361-70. http://dx.doi.org/10.1037/0894-4105.6.4.361

63. Särkämö T, Tervaniemi M, Laitinen S, Forsblom A, Soinila S, Mikkonen M, Autti T, Silvennoinen HM, Erkkilä J, Laine M, Peretz I, Hietanen M. Music listening enhances cognitive recovery and mood after middle cerebral artery 
stroke. Brain. 2008;131(Pt 3):866-76. [PMID:18287122] http://dx.doi.org/10.1093/brain/awn013

64. Altenmüller E, Marco-Pallares J, Münte TF, Schneider S. Neural reorganization underlies improvement in strokeinduced motor dysfunction by music-supported therapy. Ann N Y Acad Sci. 2009;1169:395-405.

[PMID:19673814]

http://dx.doi.org/10.1111/j.1749-6632.2009.04580.x

65. Russo NM, Nicol TG, Zecker SG, Hayes EA, Kraus N. Auditory training improves neural timing in the human brainstem. Behav Brain Res. 2005;156(1):95-103.

[PMID:15474654]

http://dx.doi.org/10.1016/j.bbr.2004.05.012

66. Meyer M, Elmer S, Baumann S, Jancke L. Short-term plasticity in the auditory system: Differential neural responses to perception and imagery of speech and music. Restor Neurol Neurosci. 2007;25(3-4):411-31. [PMID:17943016]

67. Baumann S, Koeneke S, Schmidt CF, Meyer M, Lutz K, Jancke L. A network for audio-motor coordination in skilled pianists and non-musicians. Brain Res. 2007;1161: 65-78. [PMID:17603027] http://dx.doi.org/10.1016/j.brainres.2007.05.045

68. Koelsch S. A neuroscientific perspective on music therapy. Ann N Y Acad Sci. 2009;1169:374-84. [PMID:19673812] http://dx.doi.org/10.1111/j.1749-6632.2009.04592.X

69. Schneider S, Schönle PW, Altenmüller E, Münte TF. Using musical instruments to improve motor skill recovery following a stroke. J Neurol. 2007;254(10):1339-46. [PMID:17260171] http://dx.doi.org/10.1007/s00415-006-0523-2

70. Lohse KR, Lang CE, Boyd LA. Is more better? Using metadata to explore dose-response relationships in stroke rehabilitation. Stroke. 2014;45(7):2053-58. [PMID:24867924] http://dx.doi.org/10.1161/STROKEAHA.114.004695

71. Dobkin BH, Plummer-D’Amato P, Elashoff R, Lee J; SIRROWS Group. International randomized clinical trial, stroke inpatient rehabilitation with reinforcement of walking speed (SIRROWS), improves outcomes. Neurorehabil Neural Repair. 2010;24(3):235-42. [PMID:20164411] http://dx.doi.org/10.1177/1545968309357558
72. Choi Y, Gordon J, Park H, Schweighofer N. Feasibility of the adaptive and automatic presentation of tasks (ADAPT) system for rehabilitation of upper extremity function poststroke. J Neuroeng Rehabil. 2011;8(1):42.

[PMID:21813010]

http://dx.doi.org/10.1186/1743-0003-8-42

73. Goodman RN, Rietschel JC, Roy A, Jung BC, Diaz J, Macko RF, Forrester LW. Increased reward in ankle robotics training enhances motor control and cortical efficiency in stroke. J Rehabil Res Dev. 2014;51(2):213-27. [PMID:24933720] http://dx.doi.org/10.1682/JRRD.2013.02.0050

74. Bragoni M, Broccoli M, Iosa M, Morone G, De Angelis D, Venturiero V, Coiro P, Pratesi L, Mezzetti G, Fusco A, Paolucci S. Influence of psychologic features on rehabilitation outcomes in patients with subacute stroke trained with robotic-aided walking therapy. Am J Phys Med Rehabil. 2013;92(10 Suppl 2):e16-25. [PMID:24052026] http://dx.doi.org/10.1097/PHM.0b013e3182a20a34

75. Haghgoo HA, Pazuki ES, Hosseini AS, Rassafiani M. Depression, activities of daily living and quality of life in patients with stroke. J Neurol Sci. 2013;328(1-2):87-91. [PMID:23522526] http://dx.doi.org/10.1016/j.jns.2013.02.027

Submitted for publication April 4, 2015. Accepted in revised form October 2, 2015.

This article and any supplementary material should be cited as follows:

Zondervan DK, Friedman N, Chang E, Zhao X, Augsburger R, Reinkensmeyer DJ, Cramer SC. Home-based hand rehabilitation after chronic stroke: Randomized, controlled single-blind trial comparing the MusicGlove with a conventional exercise program. J Rehabil Res Dev. 2016;53(4):457-72. http://dx.doi.org/10.1682/JRRD.2015.04.0057

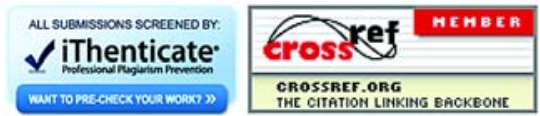


